\title{
Infection of Toxoplasma gondii in Humans and Livestock Animals: An Emerging Silent Threat for Bangladesh
}

\author{
Taibur Rahman*, Atiqur Rahman, Sajib Chakraborty \\ Department of Biochemistry and Molecular Biology, University of Dhaka, Dhaka, Bangladesh \\ Email: *taibur@du.ac.bd
}

How to cite this paper: Rahman, T., Rahman, A. and Chakraborty, S. (2018) Infection of Toxoplasma gondii in Humans and Livestock Animals: An Emerging Silent Threat for Bangladesh. Open Journal of Medical Microbiology, 8, 109-117.

https://doi.org/10.4236/ojmm.2018.84010

Received: September 12, 2018

Accepted: November 5, 2018

Published: November 21, 2018

Copyright $\odot 2018$ by authors and Scientific Research Publishing Inc. This work is licensed under the Creative Commons Attribution International License (CC BY 4.0).

http://creativecommons.org/licenses/by/4.0/

(c) (i) Open Access

\begin{abstract}
Toxoplasma gondii is an intracellular, zoonotic protozoan parasite that causes toxoplasmosis. It can potentially infect almost all mammalian and avian hosts including one-third of the human population world-wide. The major target group of the parasite includes immunocompromised patients (e.g. AIDS, cancer, organ transplantation) and fetus bearing pregnant women where it develops toxoplasmic encephalitis, myocarditis, chorioretinitis and abnormal fetal brain development or stillbirths respectively. In this review, we have presented the current status of $T$. gondii infection in livestock animals and human population in Bangladesh to assess the country-wide relative risk. Although exact prevalence is difficult to predict due to the scarcity of data, nevertheless existing literature suggests that $16 \%-39 \%$ humans and $8 \%-70 \%$ domestic animals are infected with $T$. gondii, which implies Bangladeshi population is at high risk of toxoplasmosis. Furthermore, we have proposed a potential area of research to decipher the genetic diversity and transmission routes of $T$. gondii infection into Bangladeshi population.
\end{abstract}

\section{Keywords}

Toxoplasma gondii, Seroprevalence, Livestock Animals, Humans, Bangladesh

\section{Introduction}

Toxoplasma gondii is an important zoonotic protozoan parasite that can infect any warm blooded animals including humans and causes toxoplasmosis [1]. T. gondii belongs to the group of phylum Apicomplexa that was first identified in African rodent “Ctenodactylus gundii" in 1908 by Nicolle and Manceaux at Pasteur institute of Tunis. T. gondii is widely distributed in nature and therefore in- 
fection rate varies from $10 \%$ - 90\% world-wide depending on environmental or socioeconomic factors and geographic locations [2]. Following an acute infection in healthy immunocompetent individuals, the fast replicating tachyzoite generally develops either mild flu-like symptoms and lymphadenopathy or asymptomatic clinical outcome. Importantly, active immune system can eliminate majority of $T$. gondii tachyzoite, but a portion of them undergoes developmental switching into slow replicating dormant bradyzoites particularly in skeletal muscle and brain [3] [4]. The transition from tachyzoite to bradyzoite is critical for establishing chronic and persistent infection. However, in immunocompromised patients (e.g. AIDS, organ transplantation and cancer), the parasite can be fatal by reactivation of latent infection which can cause toxoplasmic encephalitis, myocarditis and eye disease, mostly retinochoroiditis [5] [6]. In addition, pregnant women constitute special risk group, because after primary infection, the parasite can easily transmit into developing fetus and thereby causes abnormal brain development, stillbirth or even abortion of the unborn child depending on the stage of infection [7]. As T. gondii is capable of infecting both animal and human, this has major relevance on medical and veterinary health and economy [8].

T. gondii has been found to be associated with many other diseases. A case control study has confirmed the presence IgG antibodies to $T$. gondii in patients with diabetes mellitus [9]. The authors found two folds higher rate of $T$. gondii infection in diabetic patients than healthy controls. The possible reason has been identified as $T$. gondii mediated destruction of beta cells of pancreas, which, therefore, impacts on insulin secretion [9]. Contrary, another study found a lack of association of $T$. gondii with diabetes mellitus [10]. Therefore, proofing the hypothesis "chronic T. gondii infection is a risk factor for developing diabetes mellitus" needs further experimental validation. In addition, $T$. gondii infection can be associated with autism [11]. Since autism affects male more frequently than female with a ratio of 4:1 (male: female), and the presence of increased concentration of testosterone in latent toxoplasmic individual than toxoplasma negative, it may suggest the possibility of association of $T$. gondii infection with developing autism spectrum disorder in male [11] [12]. From the above point of views, $T$. gondii infection can be considered as one of the major public health problems world-wide.

\section{Life Cycle and Transmission of T. gondii}

T. gondii has two distinctive stages (sexual and asexual) in their life cycle where the sexual part is accomplished in the intestine of the definitive host "Cat" and asexual reproduction occurs in an intermediate host, for instance, livestock animals or human [13]. Sexual reproduction in cat's intestine leads to the development of $T$. gondii Oocyst which can shed into the environment through cat faeces [14] (Figure 1). Therefore, Oocysts contaminated foods and water are considered as the major transmission route of the parasite into the human [15]. 


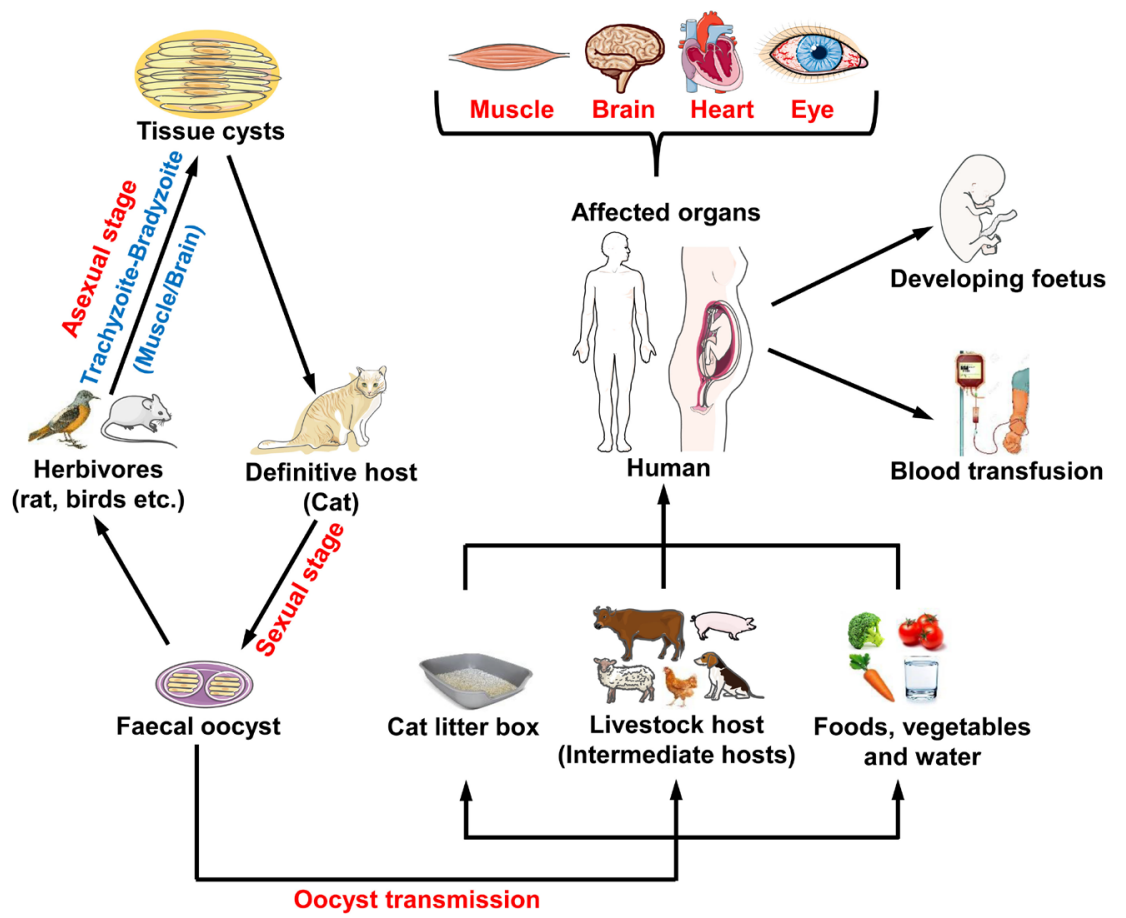

Figure 1. Life cycle and transmission of T. gondii into domestic animals and humans.

However, due to the persistence of T. gondii in muscle and brain of warm-blooded live stock animals, the parasite can transmits into human via eating raw or undercooked meats/meat products [16]. Furthermore, developing fetus can get the infection from acutely infected mother through placental transmission [17]. In addition, the parasite may also transmit through blood transfusion particularly in $\beta$-thalassemic patients who require a constant supply of blood from other individuals [18] (Figure 1).

\section{Prevalence of $T$ gondii in Major Livestock Animals and Humans of Bangladesh}

Several studies have been performed on the prevalence of $T$. gondii in livestock animals (Table 1) and humans (Table 2) in Bangladesh. For the first time, Samad and colleagues have identified the presence of anti- $T$. gondii antibodies in the blood of sheep, goats and cattle at Mymensingh [19] [20]. The authors showed that $18 \%$ sheep, $12 \%$ - 38\% goats and $16 \%$ cattle were infected with $T$. gondii. Another study conducted in 2011 by Shahiduzzaman M, has identified $T$. gondii antibodies in $40 \%$ sheep, $32 \%$ goats and $12 \%$ cattle. However, the highest infection rate was observed recently in livestock animals of Mymensingh in a study conducted by Rahman and colleagues who identified $T$. gondii antibodies in $70 \%$ sheep, $61 \%$ goats and $27 \%$ cattle [21]. The different infection rate might be due to either increasing the incidence of $T$. gondii with time or variation of test results in diagnostic approaches.

On the other hand, a very few studies has been conducted on human particularly in pregnant women of Bangladesh where the authors have identified anti-T. 
Table 1. Prevalence of T. gondii in domestic animals of Bangladesh.

\begin{tabular}{cccccc}
\hline Host animal & Study location & Sample size (N) Prevalence (\%) & Test & Reference \\
\hline Goats & Mymensingh & 15 & 38 & LAT & {$[19]$} \\
Sheep & & 17 & 18 & & \\
Goats & Mymensingh & 306 & 12 & LAT & {$[20]$} \\
Cattle & & 205 & 16 & & \\
Pigs & All over Bangladesh & 200 & 20 & HA & {$[22]$} \\
Sheep & & 15 & 40 & & \\
Goats & Mymensingh & 17 & 32 & Toxo-test MT & {$[23]$} \\
Cattle & & 22 & 12 & & \\
Sheep, & & 83 & 70 & & \\
Goats & Mymensingh & 146 & 61 & Toxo-test MT & {$[21]$} \\
Cattle & & 37 & 27 & & \\
Goats & Rajshahi & 145 & 55 & Toxo-test MT & {$[24]$} \\
Sheep & Dhaka, Mymensingh & 552 & 12 & & \\
Goats & Sirajganj, Chittagong & 300 & 16 & ELISA & {$[25]$} \\
Cattle & & 252 & 8 & & \\
\hline
\end{tabular}

LAT: Latex agglutination test, HA: Hemagglutination assay, ELISA: enzyme linked immunosorbent assay.

Table 2. Prevalence of T. gondii in humans of Bangladesh.

\begin{tabular}{ccccccc}
\hline Host & $\begin{array}{c}\text { Study } \\
\text { location }\end{array}$ & $\begin{array}{c}\text { Sample } \\
\text { size (N) }\end{array}$ & $\begin{array}{c}\text { Prevalence } \\
(\%)\end{array}$ & Test & Reference \\
\hline $\begin{array}{c}\text { Women with } \\
\text { Gyneco-Obstetrics problem }\end{array}$ & Mymensingh & 302 & 16 & LAT & {$[27]$} \\
Pregnant women & Dhaka & 286 & 39 & ELISA & {$[26]$} \\
Pregnant women & Dhaka & 46 & 33 & ELISA & {$[29]$} \\
Pregnant women & $\begin{array}{c}\text { Mymensingh, } \\
\text { Rangpur }\end{array}$ & 87 & 25 & $\begin{array}{c}\text { Toxo IgG/IgM } \\
\text { combo rapid Test }\end{array}$ & {$[30]$} \\
\hline
\end{tabular}

LAT: Latex agglutination, ELISA: enzyme linked immunosorbent assay.

gondii antibodies in the blood of $16 \%$ - 39\% of pregnant women using different serological tests (Table 2). These studies confirmed that pregnant women are more susceptible to $T$. gondii infection than non-pregnant women. Furthermore, the higher prevalence rate was observed in the age group of 31 - 45 years [23] [26]. T. gondii infection has been found to be associated with abortion (5.3\%), stillbirth $(2 \%)$ and prenatal $(<1 \%)$ death of fetus in pregnant women [27]. In addition, Ashrafunnessa and colleagues have confirmed that $T$. gondii infection was higher in poor women (53\%) compared to women with upper socioeconomic status (22\%) [26]. Recently Naheen et al. have confirmed that the rate of persistent infection ( $84 \%$ IgG) was higher than acute infection (16\% IgM) in a study on thirty nine $T$. gondii positive women [28]. Altogether these data clearly indicate the presence of $T$. gondii infection in Bangladesh. Although these studies have shown the presence of anti- $T$. gondii antibodies in the blood of mostly domestic animals and to a lesser extent in human through various serological assays (Latex agglutination, Toxo-MT test, ELISA), the more accurate 
detection technique, for instance, polymerase chain reaction (PCR) was not used for confirming the diagnosis. It can be stressed that the above-mentioned studies in human were carried out only on pregnant women, therefore the possibility of T. gondii infection on other risk groups for instance immunocompromised (HIV, cancer, transplant) or thalassemic patients has not yet been investigated.

Another limitation that seriously hindered the assessment of the country-wide prevalence of $T$. gondii infection is scarcity of country-wide data. Our analysis revealed that only the small-scale prevalence studies were conducted restricted to a certain geographical locations within Bangladesh seriously hampering the precise estimation of the actual prevalence of $T$. gondii infection in Bangladesh. For instance certain geographical locations such as Dhaka, Mymensingh, Rangpur and Rajshahi are over represented and the whole southern and north-eastern part of Bangladesh is either under-represented or missing altogether. This incomplete data makes the prevalence estimation incorrect and more importantly may lead us to underestimate the prevalence of overall $T$. gondii. It is likely that certain geographical area in Bangladesh may serve as hot-spot for $T$. gondii infections. Moreover the usage of serological assays for the diagnosis of $T$. gondii, make it prone to underestimation of $T$. gondii infection since molecular assays are required to diagnose the parasite in distinct developmental stages. Therefore, it is very difficult to conclude the exact prevalence of $T$. gondii infection in Bangladesh. However, it can be said that the infection rate may be even higher than that found by previous studies in this country and further studies on other risk groups may address this. In addition, there is no data available on the genotyping of the T. gondii strain in Bangladesh. To the end it can be concluded that further research has to be performed to find out the exact prevalence and types of $T$. gondii strain present in Bangladesh.

\section{A Possible Link between T. gondii Infection and Stillbirths/Neonatal Deaths Rates: A Grand Challenge for Bangladesh}

Bangladesh has made giant leaps toward achieving the Millennium Development Goals (MDGs). However to attain Sustainable Development Goals (SDGs), certain areas demand for urgent attention including reproductive, maternal, newborn and child health. In a recent study Abir et al. analyzing the data from the Bangladesh Demographic and Health Surveys for four years identified 29,094 cases of stillbirths and estimated rate of stillbirth in Bangladesh as 28 in 1000 births (95\% CI: 22, 34) [31]. Although the authors tried to correlate the cases of stillbirth with various social parameters such as education of mother, majority of the cases remains unexplained implying that natural causes like parasite infection can also be responsible for certain fraction of the stillbirth cases. In this circumstance, it is of utmost importance to identify the etiology of stillbirths. Therefore an epidemiological survey of parasitic infections including $T$. gondii infection may reveal the hidden association of parasite infection and stillbirth in Bangladesh. 
Although neonatal death rate has decreased significantly in Bangladesh in last 25 years (23.3 per 1000 live birth (LB) [32], the number is still high compared to other middle income countries. In this context, identifying the causes of neonatal deaths would be immensely beneficial to adopt a strategy to lower the death rates. In this context the prevalence of $T$. gondii infection in pregnant woman can enlighten to assess the risk of $T$. gondii infection for stillbirths and neonatal mortality.

\section{Prevention Strategy of T. gondii Infection}

Since $T$. gondii is mainly transmitted through eating undercooked or raw meats and meat-products, therefore the infection rate can be prevented by proper management of meat processing including use of gloves, washing cooking surface and utensils etc, ingestion of well cooked meats and meat products. Furthermore, $T$. gondii infection can be prevented by thoroughly washing oocyst contaminated foods and vegetables before eating. In addition, awareness of people particularly pregnant women could be done not to change cat litter without using proper safety. Extra precaution for instance screening of $T$. gondii infection in blood can be performed before blood transfusion. Currently, a live vaccine, S48 strain of $T$. gondii (Toxovax) is used for sheep but there is no vaccine available against $T$. gondii infection for human.

\section{Conclusion}

The current study has compiled enough evidence that indicates the presence of T. gondii infection in livestock animals and humans in Bangladesh beyond any reasonable doubt. The review also underpinned the urgent demand for using molecular approach, such as polymerase chain reaction (PCR) for confirming $T$. gondii diagnosis, to identify the strains and dissecting possible transmission routes of $T$. gondii in human and domestic animals. Such large scale molecular diagnosis based studies encompassing all the geographical regions in Bangladesh are required to estimate the true prevalence of $T$. gondii. One of the benefits of such large-scale studies includes the adaptation of potential strategies to reduce incidence and prevent the diseases associated with $T$. gondii in Bangladesh. This review is sought not only to provide the current status of $T$. gondii infection in Bangladesh and but also to encourage government and non-government organizations (NGOs) to undertake large scale studies to gain deeper insight into the association of $T$. gondii infection and stillbirths/neonatal deaths in Bangladesh.

\section{Acknowledgements}

The authors thank Department of Biochemistry and Molecular Biology, University of Dhaka for her cooperation.

\section{Conflicts of Interest}

The authors declare no conflicts of interest regarding the publication of this paper. 


\section{References}

[1] Liu, Q., Wang, Z.D., Huang, S.Y. and Zhu, X.Q. (2015) Diagnosis of Toxoplasmosis and Typing of Toxoplasma gondii. Parasites and Vectors, 8, 1-14. https://doi.org/10.1186/s13071-015-0902-6

[2] Flegr, J., Prandota, J., Sovičková, M. and Israili, Z.H. (2014) Toxoplasmosis-A Global Threat. Correlation of Latent Toxoplasmosis with Specific Disease Burden in a Set of 88 Countries. PLOS ONE, 9, e90203. https://doi.org/10.1371/journal.pone.0090203

[3] Dubey, J.P., Lindsay, D.S. and Speer, C.A. (1998) Structures of Toxoplasma gondii Tachyzoites, Bradyzoites, and Sporozoites and Biology and Development of Tissue Cysts. Clinical Microbiology Review, 11, 267. https://doi.org/10.1128/CMR.11.2.267

[4] Tenter, A.M., Heckeroth, A.R. and Weiss, L.M. (2000) Toxoplasma gondii: From Animals to Humans. International Journal for Parasitology, 30, 1217-1258. https://doi.org/10.1016/S0020-7519(00)00124-7

[5] Eza, D.E. and Lucas, S.B. (2006) Fulminant Toxoplasmosis Causing Fatal Pneumonitis and Myocarditis. HIV Medicine, 7, 415-420. https://doi.org/10.1111/j.1468-1293.2006.00393.x

[6] Saadatnia, G. and Golkar, M. (2012) A Review on Human Toxoplasmosis. Scandinavian Journal of Infectious Diseases, 44, 805-814. https://doi.org/10.3109/00365548.2012.693197

[7] Torgerson, P.R. and Mastroiacovo, P. (2013) The Global Burden of Congenital Toxoplasmosis: A Systematic Review. Bulletin of the World Health Organization, 91, 501-508. https://doi.org/10.2471/BLT.12.111732

[8] Cenci-Goga, B.T., Rossitto, P.V., Sechi, P., McCrindle, C.M.E. and Cullor, J.S. (2011) Toxoplasma in Animals, Food, and Humans: An Old Parasite of New Concern. Foodborne Pathogens and Disease, 8, 751-762. https://doi.org/10.1089/fpd.2010.0795

[9] Shirbazou, S., Delpisheh, A., Mokhetari, R. and Tavakoli, G. (2013) Serologic Detection of Anti Toxoplasma gondii Infection in Diabetic Patients. Iranian Red Crescent Medical Journal, 15, 701-703. https://doi.org/10.5812/ircmj.5303

[10] Alvarado-Esquivel, C., Loera-Moncivais, N., Hernandez-Tinoco, J., Sanchez-Anguiano, L.F., Hernandez-Madrid, G., et al. (2017) Lack of Association between Toxoplasma gondii Infection and Diabetes Mellitus: A Matched Case-Control Study in a Mexican Population. Journal of Clinical Medicine Research, 9, 508-511. https://doi.org/10.14740/jocmr3029w

[11] Flegr, J., Lindová, J. and Kodym, P. (2008) Sex-Dependent Toxoplasmosis-Associated Differences in Testosterone Concentration in Humans. Parasitology, 135, 427-431. https://doi.org/10.1017/S0031182007004064

[12] Baron-Cohen, S., Lombardo, M.V., Auyeung, B., Ashwin, E., Chakrabarti, B., et al. (2011) Why Are Autism Spectrum Conditions More Prevalent in Males? PLoS Biology, 9, e1001081.

[13] Dubey, J.P. (2009) History of the Discovery of the Life Cycle of Toxoplasma gondii. International Journal for Parasitology, 39, 877-882.

[14] Dubey, J.P., Miller, N.L. and Frenkel, J.K. (1970) The Toxoplasma gondii Oocyst from Cat Feces. The Journal of Experimental Medicine, 132, 636-662. https://doi.org/10.1084/jem.132.4.636

[15] Jones, J.L. and Dubey, J.P. (2012) Foodborne Toxoplasmosis. Clinical Infectious Diseases, 55, 845-851. https://doi.org/10.1093/cid/cis508 
[16] Cook, A.J., Gilbert, R.E., Buffolano, W., Zufferey, J., Petersen, E., et al. (2000) Sources of Toxoplasma Infection in Pregnant Women: European Multicentre Case-Control Study. British Medical Journal, 321, 142-147. https://doi.org/10.1136/bmj.321.7254.142

[17] Dunn, D., Wallon, M., Peyron, F., Petersen, E., Peckham, C., et al. (1999) Mother-to-Child Transmission of Toxoplasmosis: Risk Estimates for Clinical Counselling. The Lancet, 353, 1829-1833. https://doi.org/10.1016/S0140-6736(98)08220-8

[18] Karakas, S., Ozlem, S., Metin Tellioglu, A., Ertabaklar, H. and Ertug, S. (2012) Investigation of Anti-Toxoplasma gondii IgG and IgM Antibodies in Beta Thalaseamia Major Patients in Aydin Province. Turkish Journal of Parasitology, 36, 133-136. https://doi.org/10.5152/tpd.2012.32

[19] Samad, M.A. (1992) Serological Diagnosis of Toxoplasma gondii Associated with Abnormal Reproduction in Black Bengal Goats. Preventive Veterinary Medicine, 13, 217-220. https://doi.org/10.1016/0167-5877(92)90106-P

[20] Samad, M.A., Rahman, K.B. and Halder, A.K. (1993) Seroprevalence of Toxoplasma gondii in Domestic Ruminants in Bangladesh. Veterinary Parasitology, 47, 157-159. https://doi.org/10.1016/0304-4017(93)90186-Q

[21] Rahman, M., Azad, M.T.A., Nahar, L., Rouf, S.M.A., Ohya, K., et al. (2014) Age-Specificity of Toxoplasma gondii Seroprevalence in Sheep, Goats and Cattle on Subsistence Farms in Bangladesh. The Journal of Veterinary Medical Science, 76, 1257-1259.

[22] Biswas, H.R., Hoque, M. and Rahman, M.L. (1992) Toxoplasmosis in Pigs in Bangladesh. Asian-Australasian Journal of Animal Sciences, 6, 133-139. https://doi.org/10.5713/ajas.1993.133

[23] Shahiduzzaman, M., Islam, R., Khatun, M.M. and Batanova, T.A. (2011) Toxoplasma gondii Seroprevalence in Domestic Animals and Humans in Mymensingh District, Bangladesh. The Journal of Veterinary Medical Science, 73, 1375-1376. https://doi.org/10.1292/jvms.11-0084

[24] Rahman, M., Alauddin, M., Hossain, K.M.M., Islam, M.H., Kitoh, K., et al. (2015) Prevalence and Dynamics of Antibodies against Toxoplasma gondii in Kids Born from Naturally Infected Goats. Parasitology International, 64, 389-391. https://doi.org/10.1016/j.parint.2015.05.015

[25] Sah, R.P., Talukder, M.H., Rahman, A.K.M.A., Alam, M.Z. and Ward, M.P. (2018) Seroprevalence of Toxoplasma gondii Infection in Ruminants in Selected Districts in Bangladesh. Veterinary Parasitology: Regional Studies and Reports, 11, 1-5.

[26] Ashrafunnessa, Khatun, S., Islam, M.N. and Huq, T. (1998) Seroprevalence of Toxoplasma Antibodies among the Antenatal Population in Bangladesh. The Journal of Obstetrics and Gynaecology Research, 24, 115-119.

https://doi.org/10.1111/j.1447-0756.1998.tb00061.x

[27] Samad, M.A., Begum, N., Shamsunahar and Ahmed, M.U. (1993) Serological Diagnosis of Toxoplasma gondii Infection in Women Associated with Gyneco-Obstetric Problems. The Southeast Asian Journal of Tropical Medicine and Public Health, 24, 102-106.

[28] Naheen, C.R., Tarafder, S., Sattar, H. and Khan, S. (2016) Toxoplasma gondii Specific IgG Avidity Assay: Role and Implication in the Confirmatory Diagnosis of Acute Toxoplasmosis in Seropositive Pregnant Women. Journal of Bangabandhu Sheikh Mujib Medical University, 9, 96-99. https://doi.org/10.3329/bsmmuj.v9i2.29263

[29] Sultana, M., Hossain, M.S., Dewan, F., Sultana, J. and Rashid, M. (2014) Association 
of Toxoplasma gondii Infection with Spontaneous Abortion. Bangladesh Journal of Obstetrics and Gynecology, 29, 87-93.

[30] Margia, U.H., Begum, N., Hossain, M.S., Dey, A.R. and Alam, M.Z. (2017) Seroprevalence of Toxoplasmosis in Women in Mymensingh and Rangpur in Bangladesh: A Hospital Survey. Bangladesh Journal of Veterinary Medicine, 15, 81-85. https://doi.org/10.3329/bjvm.v15i1.34060

[31] Abir, T., Agho, K.E., Ogbo, F.A., Stevens, G.J., Page, A., et al. (2017) Predictors of Stillbirths in Bangladesh: Evidence from the 2004-2014 Nation-Wide Household Surveys. Global Health Action, 10, Article ID: 1410048. https://doi.org/10.1080/16549716.2017.1410048

[32] World Health Organization \& Bangladesh (2015) Success Factors for Women's and Children's Health: Bangladesh. World Health Organization, 47. 